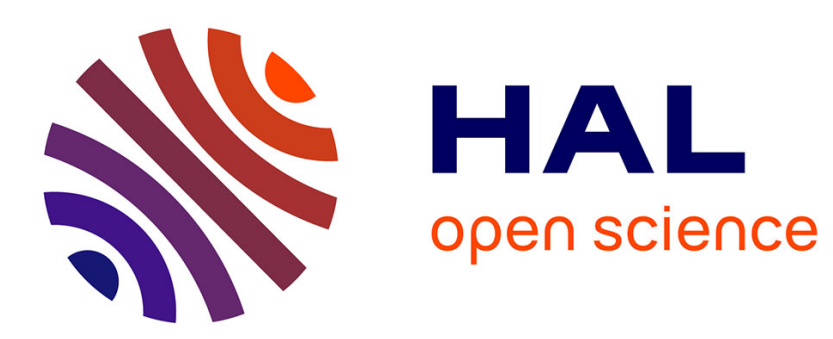

\title{
Sensitivity to solid volume fraction of gravitational instability in a granular medium
}

Fabrice Bonnet, T. Richard, P. Philippe

\section{To cite this version:}

Fabrice Bonnet, T. Richard, P. Philippe. Sensitivity to solid volume fraction of gravitational instability in a granular medium. Granular Matter, 2010, 12 (3), 9 p. 10.1007/s10035-010-0178-7 . hal-00556571

\section{HAL Id: hal-00556571 \\ https://hal.science/hal-00556571}

Submitted on 17 Jan 2011

HAL is a multi-disciplinary open access archive for the deposit and dissemination of scientific research documents, whether they are published or not. The documents may come from teaching and research institutions in France or abroad, or from public or private research centers.
L'archive ouverte pluridisciplinaire HAL, est destinée au dépôt et à la diffusion de documents scientifiques de niveau recherche, publiés ou non, émanant des établissements d'enseignement et de recherche français ou étrangers, des laboratoires publics ou privés. 


\section{Sensitivity to Solid Volume Fraction of Gravitational Instability in a Granular Medium}

Félix Bonnet

3275 Route de Cézanne, 13182 Aix en Provence cedex 5, France

Cemagref, Aix en Provence, unité OHAX

felix.bonnet@cemagref.fr

Thierry Richard

3275 Route de Cézanne, 13182 Aix en Provence cedex 5, France

Cemagref, Aix en Provence, unité OHAX

Pierre Philippe

3275 Route de Cézanne, 13182 Aix en Provence cedex 5, France

Cemagref, Aix en Provence, unité OHAX

pierre.philippe@cemagref.fr

We report experimental results on incipient dynamics of gravitational destabilization in an immersed granular medium. Two protocols have been used to reach instability: a progressive loading and a dam-break situation. The instability triggering, analyzed by high-speed imaging and CIV post-processing, reveals distinct dynamical behaviours depending on the initial packing fraction of the medium. Two destabilisation modes have been observed: a surface avalanche and a deeper phenomenon that we called circular collapse.

Gravitational instability, Avalanche, Collapse, Granular flows.

\section{Introduction}

Slope failure is a problem of high practical importance for both civil engineering structures and natural hazards management. Catastrophic events as landslides, debris flows, rock avalanches or reservoir embankment failures exemplify the potential consequences of a soil gravitational instability [1-3]. One of the most critical situation concerns a submerged sandy slope since pore pressure changes, related to groundwater seepage flow or soil dilation/contraction, can significantly affect the stability of many earth structures or natural soils [4]. 
Bonnet, F., Richard, T. and Philippe, P. (2010) Sensitivity to solid volume fraction of gravitational instability in a granular medium, Granular Matter, $12,3,317-325$. author-produced version of the final draft post-refeering

Many works have been dedicated to gravitational stability and flow of granular materials. Maximum stable slope inclination, transient destabilisation process, steady surface flows or final extent of deposit are among the major motivations of great practical interest. We can distinguish three usual situations: progressive loading of a static packing of grains up to an eventual massive destabilization, dense granular surface flow along a slope at an inclination equal or slightly above avalanche angle, and dam-break-like configuration when a granular pile is suddenly released far above the limit stability slope.

- The first category has revealed occurrence of pre-avalanche precursors due to intermittent collective grains rearrangements whose mean size and frequency progressively increase as the granular slope approaches the stability limit [5-9].

- Once the slope inclination is equal or slightly beyond the maximal stability angle, an incipient motion of grains takes place in the vicinity of the free surface. By now, only very few studies have investigated the transient regime [10-12] while many works have dealt with the steady regime of dry surface avalanche flows leading in particular to the proposal of a local granular rheology for dense flows (see [13] and references therein). According to the definition of three regimes of avalanches in fluids [14], the local rheology has been subsequently extended to the case of submarine granular flows down inclined planes [15].

- The third category corresponds to the so-called collapse or slumping which induces a fast and highly non-stationary granular flow. This dam-break configuration is achieved experimentally either by rapidly raising the retaining walls [16-19], or by removal of a confinement force [20]. Experiments [16-20] and numerical simulations $[21,22]$ have been carried out on several granular pile geometries and scaling laws have been proposed to relate the dimensions of the residual deposit to the initial aspect ratio of the pile.

All these studies have helped to gain insight into slope failures and surface flows in granular media. However, many questions remain unresolved: what are the relevant local mechanisms responsible for the precursor events and for the global instability? How does the subsequent incipient motion progressively develop? What is the role of the initial structure of the granular packing? Does the problem involve some coupled grains-fluid deformation since the dilatant-contractant behavior of the medium can cause significant pore-pressure changes [12, 23]? 
Bonnet, F., Richard, T. and Philippe, P. (2010) Sensitivity to solid volume fraction of gravitational instability in a granular medium, Granular Matter, $12,3,317-325$. author-produced version of the final draft post-refeering

the original publication is available at http://www.springerlink.com/ - DOI: 10.1007/s10035-010-0178-7

The aim of the current work is to present experimental results on the influence of the initial state on sandy slope failure. The study focuses on the two extreme configurations of the medium: densest versus loosest packing. It is shown in the following that not only the limit stability angle but also the destabilization process reveals a very sensitive dependence on solid volume fraction and, more generally speaking, on the microstructure of the medium which is significantly modified when changing the method of preparation. This sensitivity to preparation has already been underlined in dam-break situation [16] as well as in post-avalanche precursor events [9] but, to our knowledge, there has been no systematic study of this effect. In particular, in most of the previous studies on avalanche-precursors and granular collapse, initial solid volume fraction was not specified.

The paper is organized as follows. Detailed descriptions of the experimental setup and of the measurement procedure are given in section 2. Experimental observations of the solid volume fraction sensitivity in both progressive loading and dam-break configuration are presented respectively in section 3 and 4. They reveal for loose sand a somehow original destabilization process that we called circular collapse. The dam-break configuration is described more in details since it clearly highlights solid volume fraction influence. In particular, for loose sand, the transient velocity profiles are investigated and compared to previous results on avalanche triggering. Further discussions and several perspectives are drawn in section 5.

\section{Experimental setup}

The experimental setup is presented in figure 1. It is composed of a rotating rectangular cell, partially transparent, of inner dimensions $20 * 5 * 40 \mathrm{~cm}$ (length*width*height), in which a sand sample, of height $H$, is poured in water. Two water reservoirs can be used to induce upward or downward the sample a water flow which is homogenized by two layers of large glass beads ( 5 and 10mm diameter), positioned at the top and at the bottom of the cell. The sand sample lies on a fine grid placed over the lower distributor. A set of pore pressure ports has been fixed in the left lateral wall and a fast camera is used to record any movement of grains at the front wall. The cell can be rotated at small speed by a direct current motor, while the camera remains motionless. All the experiments 
Bonnet, F., Richard, T. and Philippe, P. (2010) Sensitivity to solid volume fraction of gravitational instability in a granular medium, Granular Matter, $12,3,317-325$. author-produced version of the final draft post-refeering

presented here were performed with a Hostun sand (HN 0.4/0.8) screened between 0.4 and $1 \mathrm{~mm}$, with a median diameter around $600 \mu \mathrm{m}$.

Previous experiments have been carried out on the influence of the direction and the intensity of the water flow on the stability threshold [24]. The height difference of the two water reservoirs was used to control the inner flow and to impose a hydraulic gradient $i=\Delta \tilde{P} / \rho_{W} g H$ where $\Delta \tilde{P}$ is the non-hydrostatic pressure, $\rho_{W}$ the water density $\left(\rho_{W}=10^{3} \mathrm{~kg} \cdot \mathrm{m}^{-3}\right)$ and $\mathrm{g}$ the gravitational acceleration $\left(g=9.81 m \cdot s^{-2}\right)$. The water flow remains perpendicular to the free surface and we distinguish between two regimes according to the sign of the pressure drop $\Delta \tilde{P}$ and consequently to the sign of the hydraulic gradient $i$ : a stabilizing regime $(i<0)$ when the downward global drag force induces a stabilizing contribution to the stress equilibrium of the medium and a destabilizing regime $(i>0)$ when the granular sample is partially fluidized by an upward water flow. The extreme situation when the granular bed gets completely fluidized is reached for a critical gradient $i_{c}=\Phi \frac{\rho_{g}-\rho_{W}}{\rho_{W}}$ where $\Phi$ is the solid volume fraction of the medium and $\rho_{g}$ the density of the grains $\left(\rho_{g}=2.65 \times 10^{3} \mathrm{~kg} \cdot \mathrm{m}^{-3}\right)$. From a theoretical point of view, two alternative approaches, namely discrete and continuum medium, give both the same relation between hydraulic gradient $i$ and instability angle $\theta_{\text {ins }}$ :

$$
\theta_{i n s}=\varphi-\arcsin \left(\frac{i}{i_{c}} \sin \varphi\right)(1)
$$

where $\varphi$ is whether an effective friction angle in a continuum medium approach or an average angle of the particles geometrical traps at the upper surface [25, 26]. This equation is a particular case of a general expression [4] where the seepage angle is set to zero. Note that Eq. 1 is obtained under the assumption that the instability phenomenon is invariant along the slope and, for instance, a surface avalanche can satisfy this hypothesis. Experiments have been carried out in order to check the validity of the theoretical prediction (Eq. 1) [24]. The measurements have been obtained for moderately dense packings with a solid volume fraction around 0.55 . The stability angle is measured at the occurence of an instability 
Bonnet, F., Richard, T. and Philippe, P. (2010) Sensitivity to solid volume fraction of gravitational instability in a granular medium, Granular Matter, $12,3,317-325$. author-produced version of the final draft post-refeering

which, in these experimental conditions, is a surface avalanche. As we can see in Figure 2, the theoretical prediction of the instability threshold is in good agreement with the experimental data. A large range of variation of the stability angle is observed, from $0^{\circ}$ in destabilizing condition $(i>0)$ to $90^{\circ}$ and even more in stabilizing condition $(i<0)$.

In the present study, experiments were carried out to check the influence of the initial solid volume fraction on both the instability angle and the destabilization process. The following preparation method is used to have access to a wide range of variation for the solid volume fraction. The sand is first immersed in the cell, initially vertical, and then fluidized for a few seconds. A sudden stop of fluidization produces a fast sedimentation that gives rise to a loose initial packing: $\Phi \simeq 0.52$. Then, several gentle mallet taps give can settle the packing up to $\Phi \simeq 0.59$. Then, starting from either dense or loose sand, two different protocols are used to reach gravitational instability, namely progressive loading and dam-break configuration. The experiments were performed in hydrostatic condition, with no water flow, except in the dam-break protocol where a stabilizing flow is preliminary imposed and suddenly removed so as to trigger the gravitational instability at a given inclination.

\section{Instability triggering by progressive loading}

\subsection{Influence of the solid volume fraction}

To test the influence of the initial solid volume fraction of the sand sample, a first set of experiments was carried out as follows: starting from a given solid volume fraction $\Phi$ ranging from 0.52 (loosest case) to 0.59 (densest case), a progressive loading of the sample is achieved by rotating the cell at a small rotation rate (typically $0.75 \%$ s). The instability angles reported in Figure 3 are evaluated from measurements of the rotation time until the destabilisation is visually detected. For a loose sample, a very slight destabilization is observed at small inclinations, typically $20^{\circ}$ to $30^{\circ}$. These events have a small extent in space and time which make them only barely visible to the eye. For a dense sample, a shallow avalanche eventually triggers between $40^{\circ}$ to $55^{\circ}$ and last a few seconds until complete relaxation of the slope down to a repose angle around $30^{\circ}$. For intermediate values 
Bonnet, F., Richard, T. and Philippe, P. (2010) Sensitivity to solid volume fraction of gravitational instability in a granular medium, Granular Matter, $12,3,317-325$. author-produced version of the final draft post-refeering

of the solid volume fraction, it gets difficult to distinguish between the two types of instability which appear to be directly linked.

Note in Figure 3 the surprisingly high ratio of 2.5 between the minimal and maximal value of the stability angle. Note also that not only the threshold is clearly modified by a significant state evolution but also the way the instability triggers as discussed below.

\subsection{Description and comparison of the instability events}

We now focus on the two extremes situation, namely loosest versus densest sample. To describe and compare the two associated modes of destabilizations, the triggering instability is recorded with the fast camera (Photron SA3) at resolution $1024 * 1024$, and at high speed (from 100 to 500 frames per second). Then, the velocity fields are computed by a Correlation Image Velocimetry algorithm (CIV), through the freeware DPIVsoft [27].

The destabilization in the loosest sample involve deep grain motion, in an almost half-circular zone (5 to $10 \mathrm{~cm}$ radius at maximum) mainly located in the upstream part of the slope. We propose here to call this destabilization circular collapse or collapse since the circular shape of the mobilized area is certainly ruled by the geometry of the cell and the boundary conditions. The velocity profiles observed at about $1 \mathrm{~cm}$ below the free surface are presented in Figure $4 \mathrm{a}$, during the accelerating phase, at times $\mathrm{t}=30,60$, and $90 \mathrm{~ms}$ after the triggering time which is measured with a precision of $\pm 4 \mathrm{~ms}$. Let $\mathrm{x}$ and $\mathrm{y}$ be the direction tangential and normal to free surface. The component $\mathrm{V}_{\mathrm{x}}$ along the slope is maximal at the middle of the sample and progressively increases with time while the normal component $\mathrm{V}_{\mathrm{y}}$ has significantly non zero and negative values, especially in the upstream half of the sample. The collapse has a rather short duration, about $0.3 \mathrm{~s}$, since the sample can quickly dissipate its energy and stops. The resulting deformation of the sample is limited but not negligible and significantly above the limit displacement detection of the CIV which is here about $50 \mu \mathrm{m}$. For moderately denser samples, the duration and the deformation of the collapse seem to vanish progressively but a systematic analysis would be needed, particularly to see some statistics of size, duration and number of events. Note finally that, as a collapse deforms only slightly the free surface, it will be followed by at least another or even more collapse events before a final massive failure of the slope by 
Bonnet, F., Richard, T. and Philippe, P. (2010) Sensitivity to solid volume fraction of gravitational instability in a granular medium, Granular Matter, $12,3,317-325$. author-produced version of the final draft post-refeering

avalanching. This succession of weak destabilizations seems however badly reproducible.

The densest sample remains first unperturbed while rotating the cell then a slow volume dilatation is observed inside the bulk and progressively followed by an accelerating avalanche whose exact beginning is quite hard to define. The grain motion is confined at the free surface and, thanks to a close-up in the vicinity of the free surface and adjacent to the upper left wall, we can only get a barely sufficient spatial resolution. A typical velocity profile of the avalanche in an almost stationary state is presented in Figure $4 \mathrm{~b}$ and reveals that $V_{y}$, the component of the velocity normal to the free surface, is negligible even in the upper moving layer while the tangential component $\mathrm{V}_{\mathrm{x}}$ remains much smaller than the maximal velocities reached in the loosest collapse.

To conclude, we can clearly distinguish the avalanche, confined in a shallow layer, parallel to the slope and preceded by a slow volume expansion of the sample, from the collapse which induces significantly deeper grain motion and a velocity field with an appreciable contribution perpendicularly to the free surface.

\subsection{Discussion}

We can sum up the above observations as follows: (i) the densest sand samples are perfectly stable till a global avalanche triggers near the surface at rather high slope inclinations; (ii) the loosest sand samples get at least partially destabilized at quite smaller angles by circular collapses; (iii) sand samples at an intermediate solid volume fraction are subjected to successive and badly reproducible collapses, whose intensities are yet too slight to significantly deform the free surface, until an avalanche ultimately starts at the surface.

An angle of instability that increases with solid volume fraction is not in itself a surprising, nor particularly new, result. However, a variation of the stability threshold in such proportions associated with a distinct change of the subsequent dynamics of destabilization, these are rather original observations. Thus, referring to Figure 3, we can say that the evolution of the avalanche threshold with solid volume fraction in the range $\Phi>0.55$ confirms a rather well-known trend while, to our knowledge, the mechanism of gravitational instability by collapse in a loose medium at far much smaller inclinations was neither observed nor studied so far. 
Bonnet, F., Richard, T. and Philippe, P. (2010) Sensitivity to solid volume fraction of gravitational instability in a granular medium, Granular Matter, $12,3,317-325$. author-produced version of the final draft post-refeering

the original publication is available at http://www.springerlink.com/ - DOI: 10.1007/s10035-010-0178-7

One can wonder if these collapse events are directly or indirectly related to the pre-avalanche precursors which have been previously studied [5-9]. Kabla et al. have described the pre-avalanche regime as a succession of discrete micro-failures which "correspond to the mechanical destabilization of a static structure induced by an incremental tilt, and the energy released by each event is rapidly dissipated" [9]. This definition is rather consistent with our observations except that a collapse is not a micro-failure since it has a significant spatial extent involving a huge number of grains and a coherent bulk displacement. Each experiment by Kabla et al. has been carried out on a granular packing resulting from a previous avalanche and so corresponding to a moderately dense sample and possibly related to our intermediate case (iii). But we can infer that, for a rather loose packing, the grains find it more and more difficult to dissipate the inertial energy since the force chains, which are weaker than in a denser medium, can get more easily broken, giving rise to a rather large instability in the granular bulk, temporary or perhaps even irretrievably. This interpretation is clearly consistent with our results as well as with the sensitivity of pre-avalanche regime with preparation underlined in [9].

\section{Instability triggering by dam-break protocol}

\subsection{Loosest versus densest case: same modes of destabilization}

To highlight the dynamics of the destabilization process, we have used a dambreak like protocol almost analogous to previous works [16-20] which allows us to initiate a gravitational instability at any inclination, independently of the solid volume fraction of sample. The questions to be addressed here are the following: How is the dynamics of destabilization modified when the slope inclination is increased? Are the two types of destabilization recovered in the two extreme cases, namely loosest versus densest sample?

To impose a given slope, a downward water flow is generated to apply a stabilizing force to the sample which can be consequently inclined far above its hydrostatic stability threshold. Eq. 2 gives the value of the minimal required hydraulic gradient. Then the flow is suddenly stopped and the incipient triggering instability is recorded with the fast camera. The loading path is plotted on the 
Bonnet, F., Richard, T. and Philippe, P. (2010) Sensitivity to solid volume fraction of gravitational instability in a granular medium, Granular Matter, $12,3,317-325$. author-produced version of the final draft post-refeering

instability diagram of Figure 2. To avoid any problem of reproducibility due to flow removal, particularly with loosest samples, we have used a rather large hydraulic gradient, far above the minimal flow intensity required for slope stability. Note that the stabilizing flow induces a slight consolidation of the sample and consequently affects the volume fraction but only to a very small extent, namely $\delta \Phi / \Phi \leq 1.5 \%$.

Similarly to the case of a progressive loading path, we recover two distinct destabilization dynamics according to the initial solid volume fraction: a surface avalanche for the densest sample and a roughly circular collapse for the loosest one.

Contrarily to the circular collapse achieved by progressive loading, the dam-break instability of the loosest sample at a slope angle of $60^{\circ}$ is no more a temporary destabilization but a massive failure of the sample that leads to a final deposit at a much lower inclination of about $30^{\circ}$. However, For comparison with Figure 4a, the velocity profiles observed at about $1 \mathrm{~cm}$ below the free surface at the same times $\mathrm{t}=30$, 60, and $90 \mathrm{~ms}$ after the triggering time are plotted in Figure 4c. The component $\mathrm{V}_{\mathrm{x}}$ has exactly the same shape along the surface with the maximal value obtained at the mid-slop and increasing with time. The normal component $\mathrm{V}_{\mathrm{y}}$ has also a similar behaviour in the upper part of the sample with negative values while positive ones are observed in the lower part of the slope contrarily to Figure $4 \mathrm{a}$ where $\mathrm{V}_{\mathrm{y}}$ remains negative in the bottom half of the sample. But overall, the profiles are qualitatively quite similar and differ mainly quantitatively by the magnitude of the velocity that is consistently much higher for a $60^{\circ}$ inclination compared with a slope of $20^{\circ}$. In summary, we can conclude that the kinematics of destabilization at high inclination in a very loose sample is, at least during its initial phase, analogous to the mechanism of destabilization by collapse highlighted in section 3 in the case of a progressive loading. As an example, the duration of the collapse in the case of the dam-break destabilization at $60^{\circ}$ is estimated to be 0.26 as shown in the following section.

A typical velocity profile of a surface avalanche obtained with the dam-break protocol at inclination $60^{\circ}$ is plotted in Figure $4 \mathrm{~d}$ and the corresponding velocity field is presented in Figure 5. The spatial resolution is hardly sufficient to estimate the velocity field. However, the profiles in Figures $4 \mathrm{~b}$ and $4 \mathrm{~d}$ compare qualitatively well and confirm that the mode of destabilization remains unchanged 
Bonnet, F., Richard, T. and Philippe, P. (2010) Sensitivity to solid volume fraction of gravitational instability in a granular medium, Granular Matter, $12,3,317-325$. author-produced version of the final draft post-refeering

the original publication is available at http://www.springerlink.com/ - DOI: 10.1007/s10035-010-0178-7

while increasing the angle of the slope and moving from a progressive loading to a dam-break configuration: a slow volume expansion of the bulk with a progressive motion of the grains near the free surface giving rise to a shallow avalanche.

\subsection{Further analysis of the circular collapse}

A typical velocity field in the loosest sample case destabilized by dam-break at inclination $60^{\circ}$ is presented in Figure 6 . As can be seen, the collapse instability is far from a rotational slip, commonly encountered in soil engineering, which is characterized by upper rigid body motion over a static granular bulk with a shear band localized in a thin circular border. We indeed observe in Figure 6 a significant shear in the whole deforming zone. The velocity profile computed in depth along a vertical line (i.e. perpendicular to the free surface) at about midslope, where the tangential component of the velocity is maximal (see Figure 4c) is displayed in Figure 7. Starting from the free surface, the velocity first decreases roughly linearly with depth. Then, at a depth of about $10 \mathrm{~cm}$, the velocity starts vanishing almost exponentially with depth. Note that in a thin layer near the free surface (dashed area in Figure 7), a plateau of the velocity is observed. This is due to an artefact of the software via the correlation technique: a group of about 10 to 20 grains is used to compute an Eulerian velocity contrary to the Particle Tracking technique where a Lagrangian velocity is deduced for each grain.

A flowing depth and a shear rate were identified as follows: the velocity profile was interpolated by a linear function in two different intervals, from the maximal measured velocity $\mathrm{V}_{\max }$ to respectively $50 \%$ and $10 \%$ of $\mathrm{V}_{\max }$. The slope gives therefore the shear rate $\gamma$ and the linear extrapolation to zero defines the flowing depth $\mathrm{h}$. Finally, the values obtained by both interpolations are averaged together. Time evolution of the maximal velocity, the shear rate and the flowing depth are represented in Figure 8 for loose sand at inclination $60^{\circ}$. Each of these values increases rapidly up to a kind of plateau up to $t \simeq 0.26 \mathrm{~s}$. This evolution corresponds strictly speaking to the circular collapse. Afterwards, the flow gradually loses its circular border and turns to a surface avalanche with a progressive deformation of the free surface. During this transition, the maximal velocity remains almost constant while we observe a significant decrease of the 
Bonnet, F., Richard, T. and Philippe, P. (2010) Sensitivity to solid volume fraction of gravitational instability in a granular medium, Granular Matter, 12, 3 , 317-325. author-produced version of the final draft post-refeering

flowing depth and, consequently, an increase of the shear rate. Finally, after 0.52$0.53 \mathrm{~s}$, the flow slows down until total deposition of the avalanche.

Time evolution of the velocity profile is extracted indifferently on the same vertical line for each velocity field. But as soon as the free surface inclination starts to deform, this line is no more perpendicular to it. The assumption that the vertical line remains perpendicular to the free surface is roughly true until $t \simeq 0.26 \mathrm{~s}$. Afterwards, the shape of the normal velocity profiles is almost conserved but not exactly the amplitude and, as a result, the tangential contribution increases. This zone where the circular collapse starts vanishing is represented by the grey area in Figure 8 .

\subsection{Comparison and discussion on velocity profiles}

In all cases, the comparison between dense and loose sand shows that a circular collapse is a deeper phenomenon than an avalanche $(12 \mathrm{~cm}$ maximum versus less than $1 \mathrm{~cm}$ ). In spite of the lack of spatial resolution and of the underestimation of both shear rate and maximum velocity in the dense case, we can assert that the circular collapse is faster than the avalanche. Moreover, we can infer that, contrarily to the avalanche, the circular collapse is due to boundary effects and is surely somehow controlled by the two lateral walls: motion starts at the top wall while a dead zone appears on the bottom right corner of the sample by abutment on the wall. This point should be clarified in future work.

Despite the fact that circular collapse in loose sand differs significantly from surface avalanche in dense sand, the instantaneous velocity profiles measured during the instability triggering are reminiscent to those observed in dry avalanches, indifferently in transient and steady regimes. [10, 11, 13, 28-30]. To compare and discuss more in details those velocity profiles, we will first refer to the work carried out by Courrech du Pont et al. [14] who have defined three flow regimes for a granular avalanche in a fluid based on the fall under gravity of one grain in fluid: a free fall regime for which there is no fluid influence (i.e. the classical dry regime) and two regimes where the avalanche dynamics is controlled by the fluid drag force and for which the grain motion reaches respectively a viscous and an inertial limit regime. Following their definitions of the relevant dimensionless groups, namely Stokes number and grain/fluid density ratio, our 
Bonnet, F., Richard, T. and Philippe, P. (2010) Sensitivity to solid volume fraction of gravitational instability in a granular medium, Granular Matter, 12, 3 , 317-325. author-produced version of the final draft post-refeering

experiments appear to be situated in the close vicinity of the boundary between viscous and inertial limit regime with a particle Reynolds number $\operatorname{Re} \approx 2$. But, for such values of Re close to unity in this case, we can no more use the Stokes drag force (valid only for $\mathrm{Re} \ll 1$ ) in the whole viscous regime or a constant drag coefficient (valid only for $\operatorname{Re} \gg 1$ ) in the whole inertial regime as was done in [14]. To clarify this point, we propose to connect the two limit regimes with the following empirical expression of the drag coefficient derived from [31]: $C_{d}=C_{\infty}\left(1+\alpha \mathrm{Re}^{-1 / 2}\right)^{2}$ where the parameters are typically $C \infty \approx 1$ and $\alpha \approx 5$ for a rather angular sand as the one used in our experiments. Then, with the same approach than in [14] and starting from the momentum equations of a grain falling in a fluid down a slope $\theta$, we can derived the following expression for the asymptotic Reynolds number reached in the limit regime: $\operatorname{Re}^{\infty}=\frac{\alpha^{2}}{4}\left[\sqrt{1+\frac{4}{\alpha}\left(\operatorname{Re}_{v}{ }^{\infty}\right)^{1 / 2}}-1\right]^{2}$ where $\operatorname{Re}_{v}{ }^{\infty}=\frac{4 \rho_{W}\left(\rho_{g}-\rho_{W}\right) g \sin \theta d^{3}}{3 \alpha^{2} C_{\infty} \eta_{W}{ }^{2}}$ is the final Reynolds number in the purely viscous limit regime (i.e. Stokes drag force). For grains diameters corresponding to our Hostun sand, namely $0.4<d<1.0 \mathrm{~mm}$, and with inclinations between $20^{\circ}$ and $90^{\circ}$, one gets typical Reynolds numbers in the range $10<\operatorname{Re}^{\infty}<90$ which attest that our results apply to the inertial limit regime.

With an analysis analogous to [14], the rheology obtained for dry granular flows [13] has been extended to steady granular flows down an inclined plane taking into account the influence of the interstitial fluid [15]. Specific rheological laws are achieved for the three regimes which predict in particular that the velocity profiles in both free fall and inertial limit regimes are qualitatively similar. Those predictions are no more valid for surface flows on a granular heap. In this case, several studies in heap and rotating drum configurations have indeed revealed both in free fall regime $[13,28,30,32]$ and inertial limit regime [33] that the steady velocity profiles remain located in the vicinity of the surface and are composed of an upper linear part in the flowing layer followed by a lower exponential tail that vanishes with depth in the granular bulk. A phenomenological model succeeds in accounting for the linear profile [29]. 
Bonnet, F., Richard, T. and Philippe, P. (2010) Sensitivity to solid volume fraction of gravitational instability in a granular medium, Granular Matter, $12,3,317-325$. author-produced version of the final draft post-refeering

The same kind of velocity profiles is recovered, almost partially, in the transient regime of dry granular avalanches on a heap $[10,11]$. So, following the previous observations on steady flows, it is not entirely surprising that our experiments, in the inertial limit regime, display qualitatively the same shape for the instantaneous velocity profile as can be seen in Figure 7. However, this similarity is not so obvious because, contrarily to the avalanches which are almost invariant along the slope, the circular collapse in loose sand is drastically different since, as already described ahead, it mobilizes a very deep zone, with roughly a half-disk shape (see Figure 6), which is mainly governed by the cell geometry.

We can however compare more quantitatively the velocity profile computed on the cut located in the deepest flowing layer perpendicularly to the free surface with the ones observed in dry flows $[10,11]$. In both cases, the maximal velocity at the surface agrees with the characteristic velocity of the regime [14]: $U_{f f}=\sqrt{g d}$ for the free fall regime and $U_{i}=\sqrt{\left(\rho_{g} / \rho_{W}-1\right) g d}$ for the inertial limit regime. Here, since $\bar{d} \approx 600 \mu \mathrm{m}$, one gets $U_{i} \approx 9 \mathrm{~cm} \cdot \mathrm{s}^{-1}$ which gives actually the good order of magnitude (see Figure 7). The main difference lies in the depth of the flowing zone which goes up to about $10 \mathrm{~cm}$ for the circular collapse when it remains equal to only three times the grain diameter in [11]. Nearly the same characteristic length of the exponential tail is observed in [10] with, above this transition region of the velocity profile, a linear part that progressively extends in depth up to a maximal flow thickness. It has been shown that the size of this flowing layer is governed by the channel width and the friction on the sidewalls $[13,30,32]$. In [11], it appears that the avalanche duration is too small for the linear part to be observed in the velocity profile. But this is obviously not the case in our experiments where the maximal flow depth should therefore logically be ruled by sidewalls friction and cell width, namely $W=5 \mathrm{~cm}$. In our set-up we do not know the value of friction at the sidewalls $\mu_{W}$, but using the value obtained in [30], one gets for the flowing thickness at inclination $60^{\circ}: \frac{h}{W}=\frac{\tan \theta-\tan \theta_{i n s}}{\mu_{W}} \approx 2$ which is in good agreement with the experimental velocity profile shown in Figure 7 and with the transient evolution of the flowing depth presented in Figure $8 c$. 
Bonnet, F., Richard, T. and Philippe, P. (2010) Sensitivity to solid volume fraction of gravitational instability in a granular medium, Granular Matter, 12, 3 , 317-325. author-produced version of the final draft post-refeering

the original publication is available at http://www.springerlink.com/ - DOI: 10.1007/s10035-010-0178-7

To conclude on the velocity profiles and as already noted in sections 3.2 and 4.1, the spatial resolution is too bad in our experiments to compute perfectly reliable profiles for the surface avalanches in dense sand. However, we expect to observe approximately the same as in dry transient avalanches $[10,11]$.

\section{Conclusion and prospects}

The gravitational instability experiments by progressive loading have revealed a high sensitivity to solid volume fraction of both the instability angle, which is modified in very large proportions, and the subsequent dynamics of destabilization: dense surface avalanche versus loose circular collapse. A question that will require attention is whether the mechanism of destabilization by collapse presents or not strong analogies with the pre-avalanche precursor events.

In previous studies on dam-break configurations, the influence of the initial solid volume fraction has not been discussed at all despite the sensitivity to preparation has been already reported before [16]. Yet this influence is very clearly underlined in our preliminary experiments presented in section 4 and a more detailed analysis is in progress.

Following the preliminary results presented in this paper, a more systematic study on sensitivity of gravitational instability triggering to preparation will be carried out in both progressive loading and dam-break configurations. Note that even if the solid volume fraction is used as a control parameter it reflects only partially the complexity of the granular microstructure from which originate the local mechanisms responsible for the micro-failures described in [5-9]. Other quantities should be more relevant than the solid volume fraction as for instance the dilatancy angle $[12,23]$ or the mean coordination number. Moreover, to analyze the role of boundary conditions, we will vary the geometry of the cell by changing independently of each other the width, height and depth of the sample. In addition to the experiments, a DEM simulation will be used in order first to test in detail this influence of the microstructure on instability threshold and subsequent incipient dynamics and second to check the relevance of a specific failure criterion, namely the second order work sign (see [34] and references therein), to 
Bonnet, F., Richard, T. and Philippe, P. (2010) Sensitivity to solid volume fraction of gravitational instability in a granular medium, Granular Matter, $12,3,317-325$. author-produced version of the final draft post-refeering

the original publication is available at http://www.springerlink.com/ - DOI: 10.1007/s10035-010-0178-7

discriminate between shear localization in dense sand and diffuse instability in loose sand.

\section{Acknowledgements}

This work was supported by the ANR project MONHA. The authors wish to thank Olivier Pouliquen, Maxime Nicolas and François Nicot for stimulating discussions.

\section{References}

[1] Iverson, R.M.: The physics of debris flows. Rev. Geophys. 35(3), 245-296 (1997).

[2] Hampton, M.A., Lee, H.J., Locat, J.: Submarine landslides. Rev. Geophysics 34(1), 33-59 (1996).

[3] Hewitt, K., Clague, J.J., Orwin, J.F.: Legacies of catastrophic rock slope failures in mountain landscapes. Earth Sci. Rev. 87, 1-38 (2008).

[4] Iverson, R.M., Major, J.J.: Groundwater seepage vectors and the potential for hillslope failure and debris flow mobilization. Water Res. Res. 22(11), 1543-1548 (1986).

[5] Staron, L., Vilotte, J.-P., Radjai, F.: Preavalanche instabilities in a granular pile. Phys. Rev. Lett. 89, 204302 (2002).

[6] Staron, L., Radjai, F.: Friction versus texture at the approach of a granular avalanche. Phys. Rev. E 72, 041308 (2005).

[7] Staron, L., Radjai, F., Vilotte, J.-P.: Multi-scale analysis of the stress state in a granular slope in transition to failure. Eur. Phys. J. E 18, 311-320 (2005).

[8] Nerone, N., Aguirre, M.A., Calvo, A., Bideau, D., Ippolito, I.: Instabilities in slowly driven granular packing. Phys. Rev. E 67, 011302 (2003).

[9] Kabla, A., Debrégeas, G., di Meglio, J.-M., Senden, T.J.: X-ray observation of micro-failures in granular piles approaching an avalanche. Europhys. Lett. 71(6), 932-937 (2005).

[10] Jop, P., Forterre, Y., Pouliquen, O.: Initiation of granular surface flows in a narrow channel. Phys. Fluids 19, 088102 (2007).

[11] Courrech du Pont, S., Fischer, R., Gondret, P., Perrin, B., Rabaud, M.: Instantaneous velocity profiles during granular avalanches. Phys. Rev. Lett. 94, 048003 (2005).

[12] Pailha, M., Nicolas, M., Pouliquen, O.: Initiation of underwater granular avalanches:

Influence of the initial volume fraction. Phys. Fluids 20, 111701 (2008).

[13] GdR MiDi: On dense granular flows. Eur. Phys. J. E 14, 341-365 (2004).

[14] Courrech du Pont, S., Gondret, P., Perrin, B., Rabaud, M.: Granular avalanches in fluids. Phys. Rev. Lett. 90, 044301 (2003).

[15] Cassar, C., Nicolas, M., Pouliquen, O.: Submarine granular flows down inclined planes. Phys. Fluids 17, 103301 (2005).

[16] Daerr, A., Douady, S.: Sensitivity of granular surface flows to preparation. Europhys. Lett. 47(3), 324-330 (1999).

[17] Lajeunesse, E., Monnier, J.B., Homsy, G.M.: Granular slumping on a horizontal surface. Phys. Fluids 17, 103302 (2005). 
Bonnet, F., Richard, T. and Philippe, P. (2010) Sensitivity to solid volume fraction of gravitational instability in a granular medium, Granular Matter, 12, 3 , 317-325. author-produced version of the final draft post-refeering

the original publication is available at http://www.springerlink.com/ - DOI: 10.1007/s10035-010-0178-7

[18] Lube, G., Huppert, H.E., Sparks, R.S.J., Hallworth, M.A.: Axisymmetric collapse of granular columns. J. Fluid Mech. 508, 175-199 (2004).

[19] Balmforth, N.J., Kerswell, R.R.: Granular collapse in two dimensions. J. Fluid Mech. 538, 399-428 (2005).

[20] Siavoshi, S., Kudrolli, A.: Failure of a granular step. Phys. Rev. E 71, 051302 (2005).

[21] Lacaze, L., Kerswell, R.R.: Axisymmetric granular collapse: A transient 3D flow test of viscoplasticity. Phys. Rev. Lett. 102, 108305 (2009).

[22] Staron, L., Hinch, E.J.: Study of the collapse of granular columns using two-dimensional discrete-grain simulation. J. Fluid Mech. 545, 1-27 (2005).

[23] Iverson, R.M.: Regulation of landslide motion by dilatancy and pore pressure feedback. J. Geophys. Res. 110, F02015 (2005).

[24] Philippe, P., Richard, T.: Start and stop of an avalanche in a granular medium subjected to an inner water flow. Phys. Rev. E 77, 041306 (2008).

[25] Albert, A., Albert, I., Hornbaker, D., Schiffer, P., Barabasi, A.-L.: Maximum angle of stability in wet and dry spherical granular media. Phys. Rev. E 56, R6271 (1997).

[26] van Rhee, C., Bezuijen, A.: Influence of seepage on stability of sandy slope. J. Geotech. Eng.

118, 1236-1240 (1992).

[27] Meunier, P., Leweke, T.: Analysis and treatment of errors due to high velocity gradients in particle image velocimetry. Exp. Fluids 35(5), 408-421 (2003).

[28] Bonamy, D., Daviaud, F., Laurent, L.: Experimental study of granular surface flows via a fast camera: A continuous description. Phys. Fluids 14(5), 1666-1673 (2002).

[29] Rajchenbach, J.: Dense, rapid flows of inelastic grains under gravity. Phys. Rev. Lett. 90, 144302 (2003).

[30] Taberlet, N., Richard, P., Valance, A., Losert, W., Pasini, J.M., Jenkins, J.T., Delannay, R.: Superstable granular heap in a thin channel. Phys. Rev. Lett. 91, 264301 (2003).

[31] Ferguson, R.I., Church, M.: A simple universal equation for grain settling velocity. J.

Sedimentary Research (2004).

[32] Jop, P., Forterre, Y., Pouliquen, O.: Crucial role of side walls for granular surface flows: consequences for the rheology. J. Fluid Mech. 541, 167-192 (2005).

[33] Jain, N., Ottino, J.M., Lueptow, R.M.: Effect of interstitial fluid on a granular flowing layer.

J. Fluid Mech. 508, 23-44 (2004).

[34] Laouafa, F., Darve, F.: Modelling of slope failure by a material instability mechanism. Comp.

Geotech. 29, 301-325 (2002). 
Bonnet, F., Richard, T. and Philippe, P. (2010) Sensitivity to solid volume fraction of gravitational instability in a granular medium, Granular Matter, $12,3,317-325$. author-produced version of the final draft post-refeering

the original publication is available at http://www.springerlink.com/ - DOI: 10.1007/s10035-010-0178-7

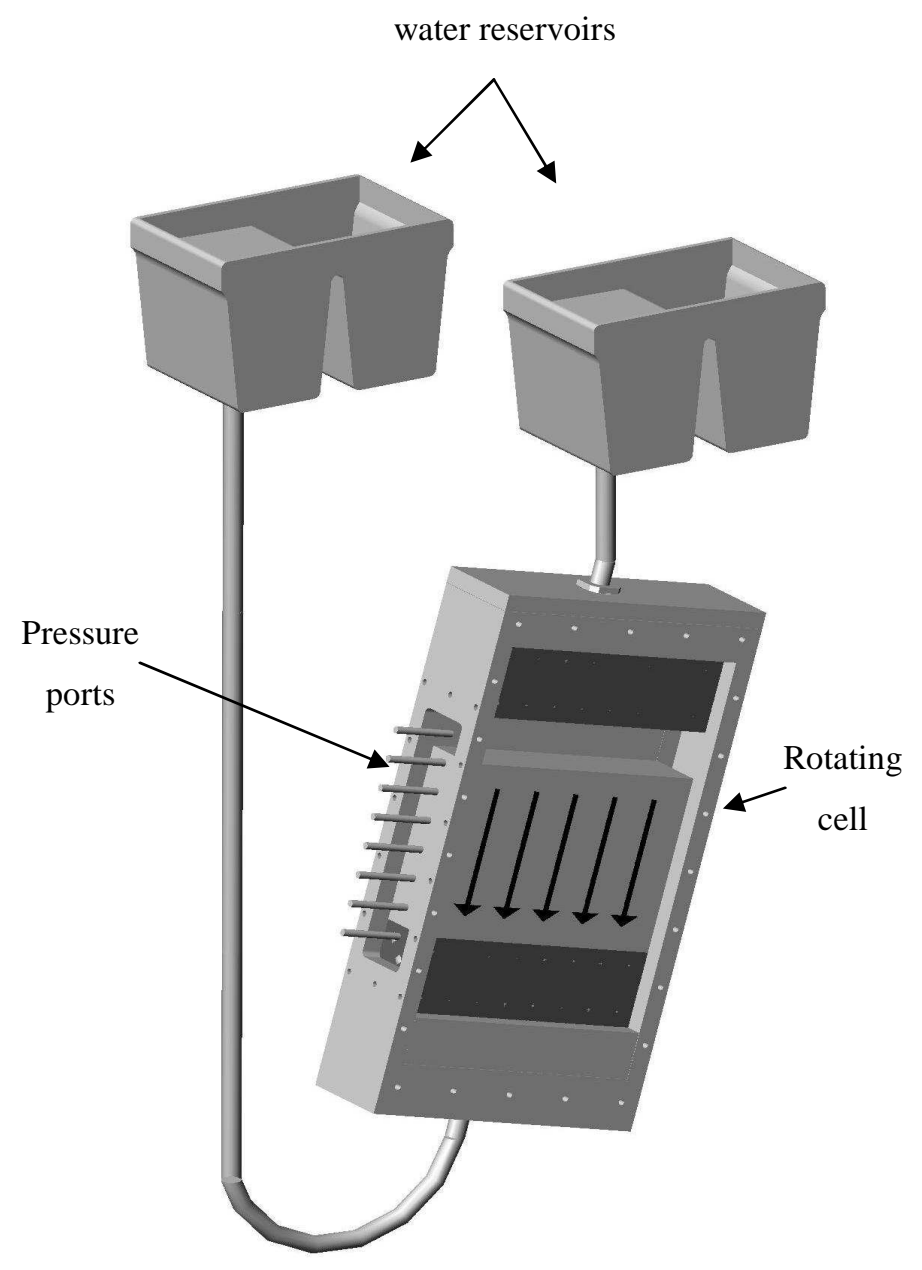

Figure 1. Diagram of the experimental setup consisting of two constant level tanks and a rotating cell with a set of pore pressure ports. The black arrows depict the seepage streamlines, upward in the present configuration and parallel to the lateral walls. 
Bonnet, F., Richard, T. and Philippe, P. (2010) Sensitivity to solid volume fraction of gravitational instability in a granular medium, Granular Matter, $12,3,317-325$. author-produced version of the final draft post-refeering

the original publication is available at http://www.springerlink.com/ - DOI: 10.1007/s10035-010-0178-7

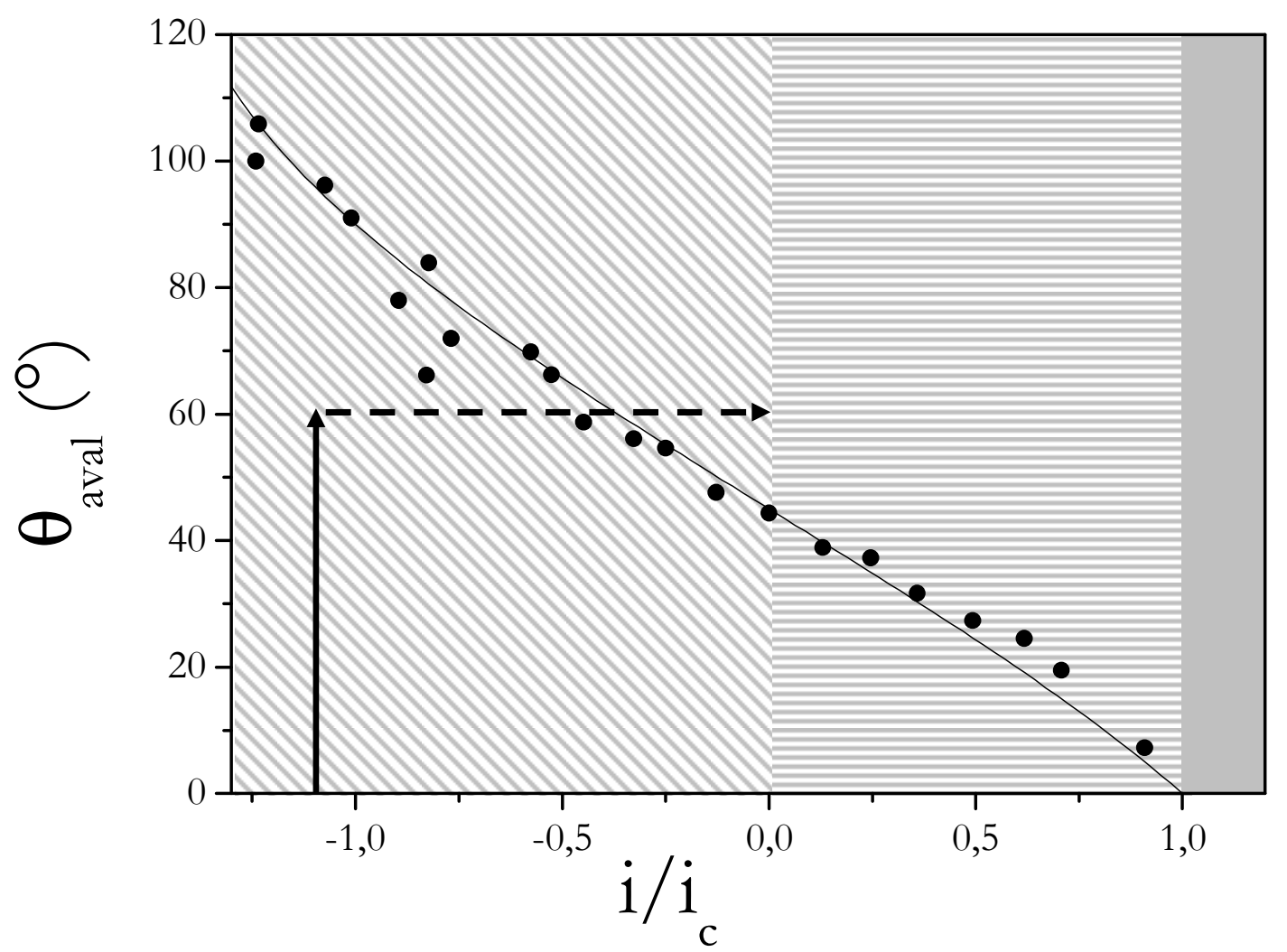

Figure 2. Plot of the avalanche angle as a function of the hydraulic gradient ratio $\mathrm{i} / \mathrm{i}_{\mathrm{c}}$ in moderately dense sand samples $\left(\Phi \approx 0.55\right.$ and $\left.i_{c} \approx 0.95\right)$. The solid line corresponds to the theoretical prediction given by Eq. 2. The arrows represent a typical loading path followed in the dam-break protocol (section 4.2). Stabilizing, destabilizing and fluidized regimes are represented respectively by diagonally hachured, horizontally hachured and plain grey area.

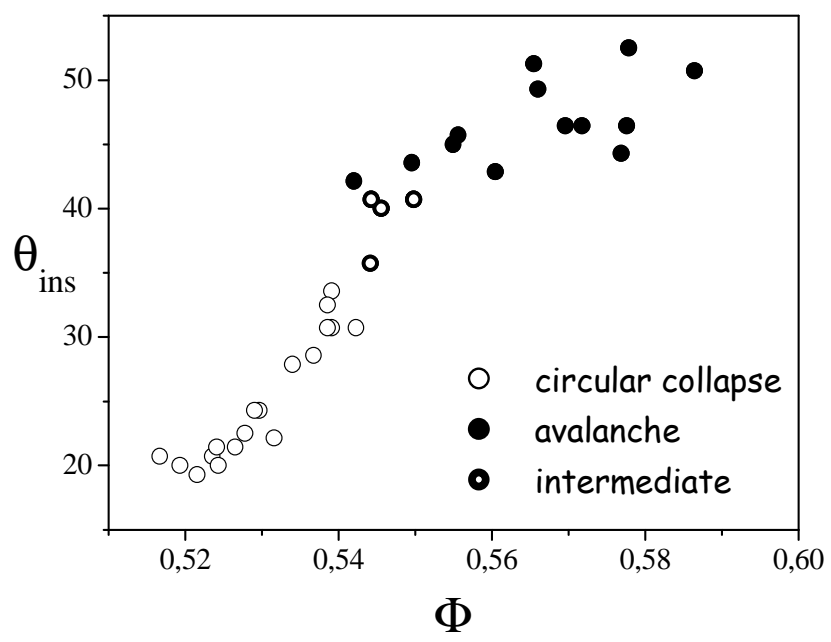

Figure 3. Maximal angle of stability versus solid volume fraction. Circular collapses are observed below 0.54 and avalanches above 0.55 with an intermediate behavior in the middle range. 
Bonnet, F., Richard, T. and Philippe, P. (2010) Sensitivity to solid volume fraction of gravitational instability in a granular medium, Granular Matter, $12,3,317-325$. author-produced version of the final draft post-refeering

the original publication is available at http://www.springerlink.com/ - DOI: 10.1007/s10035-010-0178-7
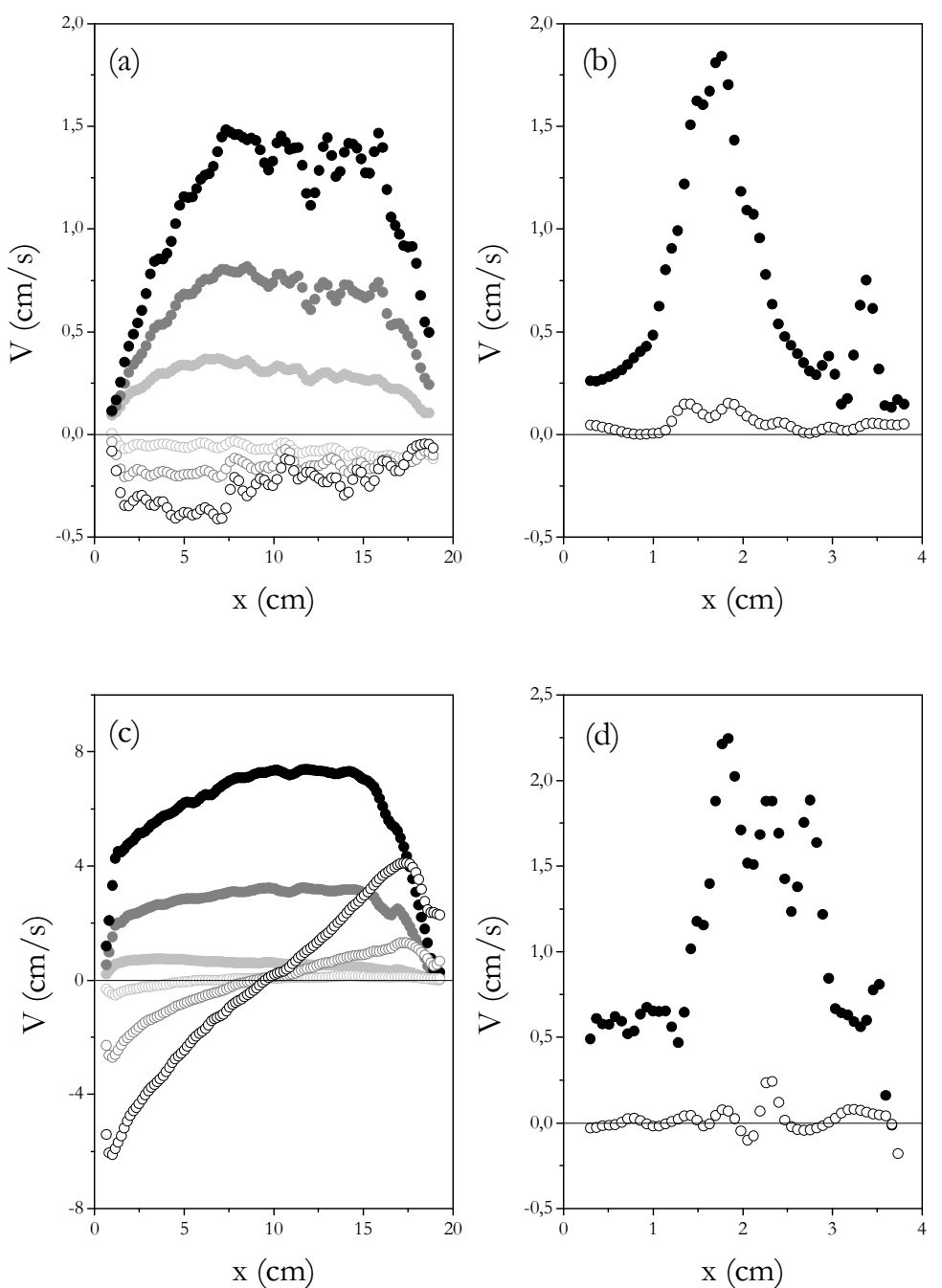

Figure 4. Velocity profiles of the tangential (closed symbols) and normal (open symbols) component along the slope, at about $1 \mathrm{~cm}$ depth below the free surface, at times $\mathrm{t}=30 \mathrm{~ms}$ (light gray symbols), $\mathrm{t}=60 \mathrm{~ms}$ (grey symbols), and $\mathrm{t}=90 \mathrm{~ms}$ (black symbols) after triggering for (a) instability at $\theta=20.2^{\circ}$ of the loosest sample by progressive loading, and (c) instability at $\theta=60^{\circ}$ of the loosest sample by dam-break protocol. Typical velocity profiles of the tangential (black closed symbols) and normal (black open symbols) component along the slope, very close to the free surface, for (b) instability at $\theta=45.0^{\circ}$ of the densest sample by progressive loading, and (d) instability at $\theta=60^{\circ}$ of the densest sample by dam-break protocol. 
Bonnet, F., Richard, T. and Philippe, P. (2010) Sensitivity to solid volume fraction of gravitational instability in a granular medium, Granular Matter, 12, 3 , 317-325. author-produced version of the final draft post-refeering

the original publication is available at http://www.springerlink.com/ - DOI: 10.1007/s10035-010-0178-7

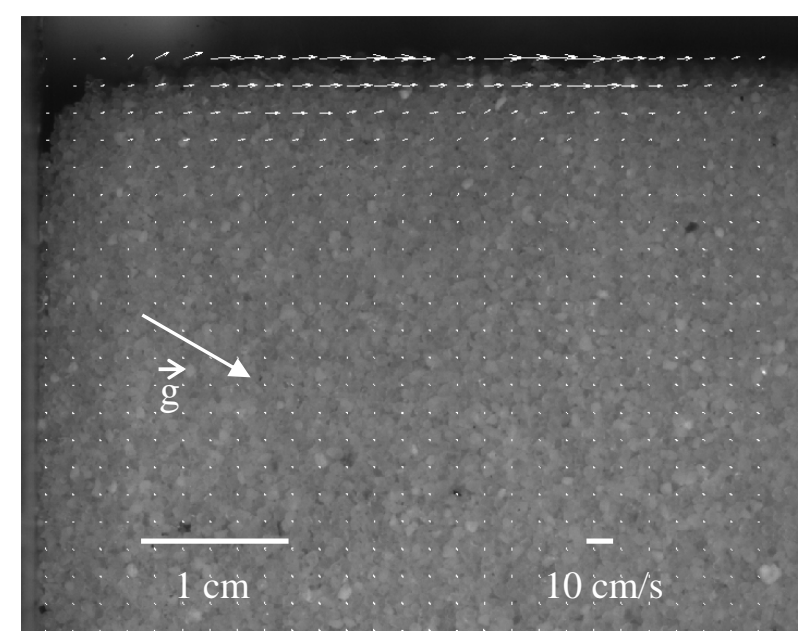

Figure 5. Typical velocity field of a surface avalanche in the densest sand sample obtained by dam-break protocol at inclination $60^{\circ}$. The frame of the image is centered on the upper left corner of the sample and is about $4 \mathrm{~cm}$ by $4.5 \mathrm{~cm}$

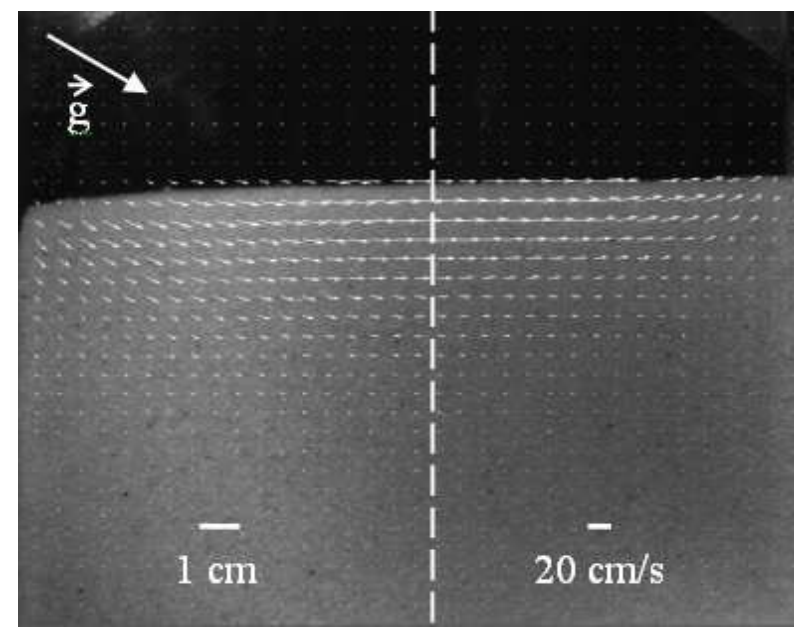

Figure 6. Typical velocity field of a circular collapse in the loosest sand sample obtained by dambreak protocol at inclination $60^{\circ}$. The vertical dash line stands for the location where the velocity profiles are computed in Figure 6. 
Bonnet, F., Richard, T. and Philippe, P. (2010) Sensitivity to solid volume fraction of gravitational instability in a granular medium, Granular Matter, 12, 3, 317-325. author-produced version of the final draft post-refeering

the original publication is available at http://www.springerlink.com/ - DOI: 10.1007/s10035-010-0178-7

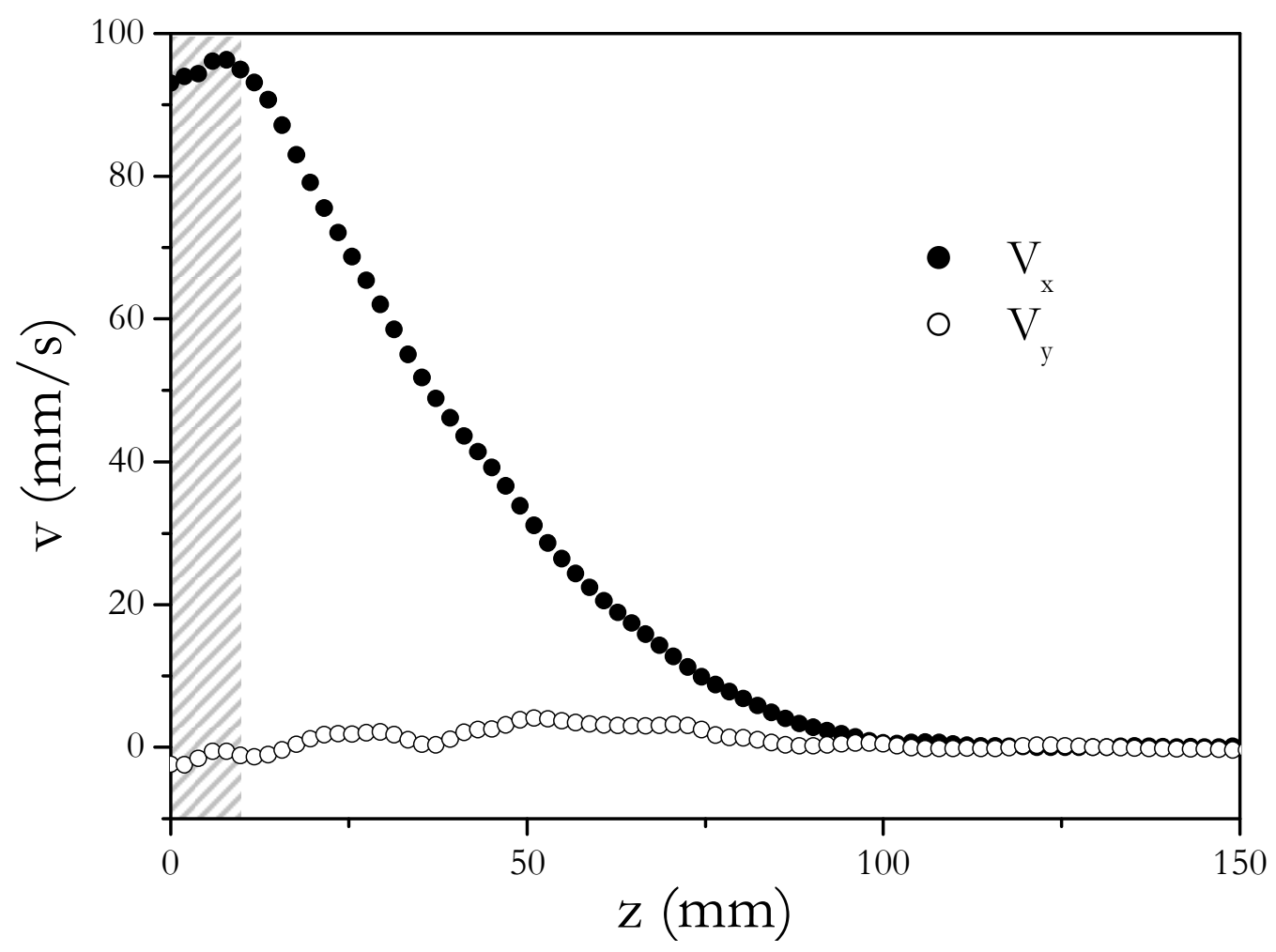

Figure 7. Typical normal $\left(\mathrm{V}_{\mathrm{x}}\right)$ and tangential $\left(\mathrm{V}_{\mathrm{y}}\right)$ velocity profiles computed along the cut represented by the dash line in Figure 5. 
Bonnet, F., Richard, T. and Philippe, P. (2010) Sensitivity to solid volume fraction of gravitational instability in a granular medium, Granular Matter, 12, 3, 317-325. author-produced version of the final draft post-refeering

the original publication is available at http://www.springerlink.com/ - DOI: 10.1007/s10035-010-0178-7
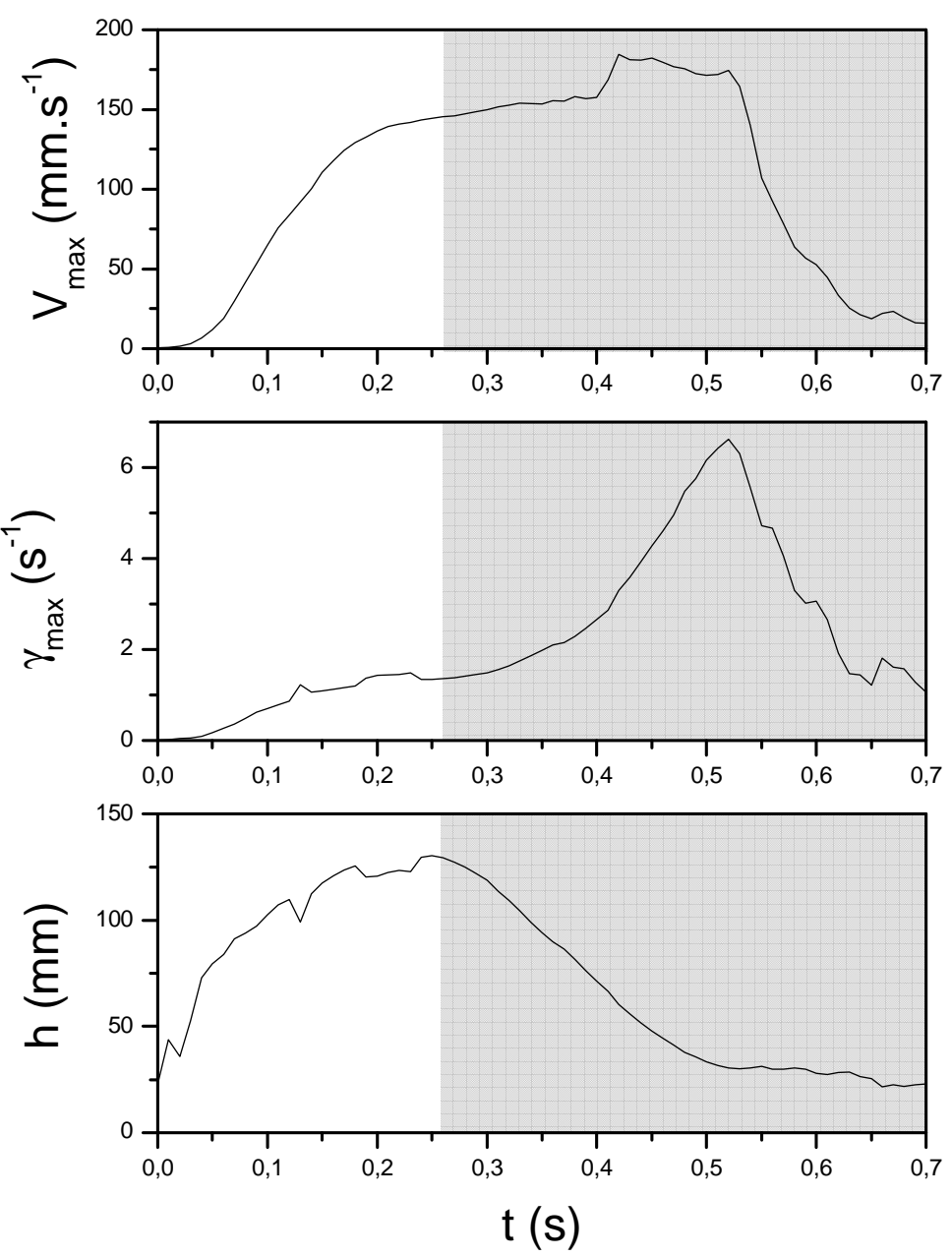

Figure 8. Time evolution of (a) the maximal velocity, (b) the shear rate and (c) the flowing depth for a loose packing $(\Phi=0.52)$ at inclination $60^{\circ}$. In shading, the cut is not anymore perpendicular to the free surface and tangential velocity contribution is no more negligible. 